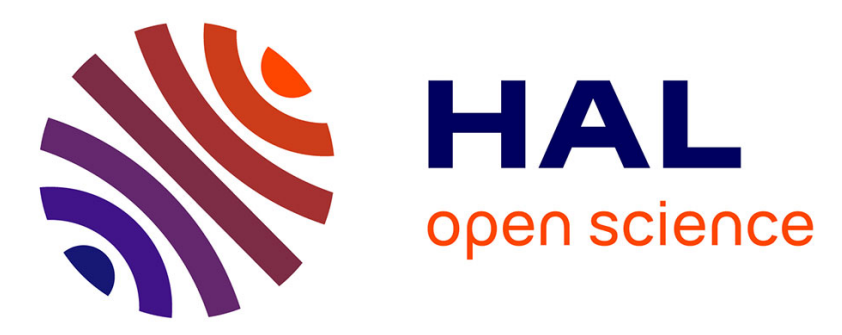

\title{
Les relations professionnelles au prisme de l'innovation: enjeux pratiques et théoriques
}

\author{
Alexandra Garabige, Frédéric Rey, Catherine Vincent
}

\section{To cite this version:}

Alexandra Garabige, Frédéric Rey, Catherine Vincent. Les relations professionnelles au prisme de l'innovation: enjeux pratiques et théoriques. La Revue de l'IRES, 2012, n 75, pp. 3-14. 10.3917/rdli.075.0003 . halshs-01771000

\section{HAL Id: halshs-01771000 \\ https://shs.hal.science/halshs-01771000}

Submitted on 19 Apr 2018

HAL is a multi-disciplinary open access archive for the deposit and dissemination of scientific research documents, whether they are published or not. The documents may come from teaching and research institutions in France or abroad, or from public or private research centers.
L'archive ouverte pluridisciplinaire HAL, est destinée au dépôt et à la diffusion de documents scientifiques de niveau recherche, publiés ou non, émanant des établissements d'enseignement et de recherche français ou étrangers, des laboratoires publics ou privés. 


\title{
Les relations professionnelles au prisme de l'innovation : enjeux pratiques et théoriques
}

\author{
Alexandra GARABIGE, Frédéric REY et Catherine VINCENT
}

Ce numéro de La Revue de l'IRES propose une sélection d'articles issus de communications présentées dans le réseau « Relations professionnelles » ${ }^{1}$ lors du Congrès «Création et innovation » de l'Association française de sociologie de juillet $2011^{2}$. Ces articles placent les régulations collectives des relations d'emploi au cœur de leur réflexion et interrogent la pertinence de la notion d'innovation pour analyser les relations professionnelles. Appréhendée initialement au travers des changements technologiques puis progressivement diffusée à de nombreux champs (développement social, santé, entreprise...), l'innovation, parce qu'elle renvoie à des activités et des domaines extrêmement variés, constitue une réalité polymorphe. Définie de manière différente selon qu'elle est portée par des experts, des économistes, des sociologues ou des politiques, c'est justement la plasticité de la notion qui a fait son succès. Au-delà de la diversité des auteurs qui l'utilisent, nous appréhendons ici l'innovation comme l'introduction d'une idée nouvelle qui, mise en œuvre, doit permettre l'amélioration d'une situation jugée insatisfaisante. Elle suppose donc un processus de réappropriation mais aussi un résultat permettant de pouvoir la qualifier d'innovation. Réfléchir

1. Le réseau thématique RT18 "Relations professionnelles " a été créé en 2007. II regroupe des chercheurs de disciplines différentes (sociologie, droit, économie, sciences politiques...) et ses activités sont ouvertes aux acteurs des relations professionnelles (http://rt18.hypotheses.org/).

2. Le congrès, intitulé "Création \& innovation », invitait plus d'un millier de sociologues à présenter leurs travaux, à discuter et à interroger ces notions, les concepts associés, leurs apports et limites dans la quarantaine de réseaux thématiques de l'Association. Dans cet ensemble, le réseau thématique RT18 a traité les innovations du point de vue des règles et des régulations collectives du travail et de l'emploi. II s'est intéressé aux acteurs, aux espaces, aux objets et aux processus de régulation pour mieux comprendre les recompositions à l'œuvre dans le champ des normes sociales d'emploi. 
à l'innovation dans le champ des relations professionnelles s'avère donc délicat, particulièrement dans une période marquée par la transformation et la remise en question des modèles sociaux. Dans le même temps, l'enjeu s'avère stimulant puisqu'il permet d'analyser si les transformations et changements en cours peuvent ou non s'imposer comme des innovations.

Partant de ces questions, l'appel à communication de 2011 a recueilli plus d'une trentaine de réponses. Les articles qui sont réunis dans ce numéro prolongent six des communications faites à l'occasion du congrès. Ces travaux de recherche portent sur des acteurs engagés dans des dynamiques de changement, parfois appelées innovations, parfois juste considérées comme innovantes. Ils font écho à un environnement en profonde mutation qui caractérise le système de relations professionnelles. Tous les aspects travaillés lors du congrès de Grenoble et toutes les dimensions de l'innovation n'ont cependant pas pu être réunis dans ce numéro. Les innovations technologiques ou techniques, la diffusion des modèles sociaux dans l'espace international ou encore les effets de la loi du 20 août 2008 " portant rénovation de la démocratie sociale » et réformant la représentativité syndicale ont donné lieu à de riches discussions en séance mais ne sont pas abordés dans ce numéro.

\section{Les relations professionnelles saisies par l'innovation}

Confronter la thématique de l'innovation à l'univers des relations professionnelles peut étonner si l'on associe les organisations syndicales, patronales et l'État à des institutions centenaires qui semblent bien éloignées de nos représentations courantes de l'innovation. L'importance des institutions et des règles, le fonctionnement de la démocratie sociale, les équilibres politiques internes et externes et le temps long des négociations conduisent à des mutations progressives. Les compromis sociaux nés à la fin de la Deuxième Guerre mondiale ont solidement ancré les régulations du travail et de l'emploi dans la négociation collective, et l'on parlera davantage de « changements institutionnels lents » (Mahoney, Thelen, 2010) que d'innovations. Les travaux en relations professionnelles s'intéressent d'ailleurs plus souvent à la reproduction ou aux mutations systémiques qu'aux innovations.

Mais si l'on reprend la définition large donnée dans le début du texte, la thématique de l'innovation interpelle le champ des relations professionnelles. Elle pose de nombreuses questions relatives à la capacité des acteurs sociaux à inventer de nouvelles pratiques, règles, relations et modalités de régulation d'abord, à les considérer comme bénéfiques ensuite, à les diffuser enfin lorsqu'elles sont jugées pertinentes. 


\section{I.1. L'innovation comme condition des mutations des relations professionnelles}

Associée à une amélioration, l'innovation pose la question du rapport de force entre les acteurs et de ce qui est considéré comme bénéfique, avec des jugements variables selon les points de vue. Une innovation patronale ou managériale peut être considérée comme une régression pour les salariés, tout comme une évolution législative offrant des possibilités d'innovation syndicale peut être perçue comme une menace pour les employeurs. La convergence des appréciations n'est certainement pas une évidence. La diffusion d'une invention ou d'une nouvelle procédure est donc tout autant problématique. Les organisations syndicales, patronales et l'État sont de grands ensembles organisationnels et il faut du temps pour qu'une pratique ou une logique nouvelle soit diffusée et réappropriée dans toutes ses ramifications. Sur le terrain, les acteurs sociaux expérimentent, tâtonnent, inventent de nouvelles formes de dialogue, de nouvelles manières de se faire entendre, de contester ou de créer de nouvelles alliances. La mutualisation de ces expérimentations sociales n'est pas automatique et souvent l'inventivité locale peine à se diffuser dans les organisations. En même temps, l'innovation apparait régulièrement dans le champ des relations professionnelles au détour d'une loi, d'une pratique ou d'une opportunité, à tel point qu'on peut se demander s'il existe un impératif systémique qui pousse les acteurs à innover. Les mutations du système de relations professionnelles engagées dès la fin des années 1970 ont par exemple fait de l'entreprise un lieu autonome de production des normes d'emploi, alors que les cadres antérieurs - la branche professionnelle, le cadre national - sont contestés par l'expansion de la mondialisation. Dans le même temps, les acteurs sociaux sont de plus en plus associés à l'action publique, cette dernière étant de plus en plus négociée (Groux, 2005). Les acteurs se retrouvent ainsi pris dans des processus d'innovations ou d'ajustements permanents. Le changement et l'innovation semblent alors être devenus une condition du renouveau syndical après des décennies de crise du syndicalisme, tandis que le paradigme socio-productif dans lequel ces acteurs se sont construits et développés depuis un siècle continue de se métamorphoser.

L'enjeu de ce numéro est de montrer comment les acteurs sociaux parviennent, tant bien que mal, à renouveler leurs pratiques et leurs approches pour continuer à réguler collectivement le travail et l'emploi, dans un environnement en mutation, marqué par l'instabilité et l'incertitude. Pour les acteurs engagés dans ces nouvelles dynamiques, il s'agit de garder le contrôle et la légitimité à intervenir dans la définition des règles du travail et de l'emploi, d'assurer ou de renforcer leurs positions dans des rapports de force qui s'expriment à des échelles de plus en plus diversifiées et face à un nombre croissant d'acteurs. 
Depuis le début des années 1980, l'architecture du système de relations professionnelles connaît des aménagements successifs, globalement en faveur du capital et des entreprises. La hiérarchie des normes et le principe de faveur, deux éléments structurants du système, sont remis en cause. Ils garantissaient pourtant un certain ordre dans les régulations : le contenu des textes négociés à un niveau ne pouvait être moins favorable aux salariés que le contenu des textes négociés à un niveau supérieur; dans l'entreprise, les accords étaient automatiquement plus protecteurs et favorables aux salariés que ce qui était prévu par la convention de branche. Cette libéralisation et la décentralisation des régulations vers les territoires et, surtout, vers les entreprises, permettent alors à ces dernières de s'affranchir plus facilement des cadres supérieurs. Parallèlement, de plus en plus de lois renvoient l'application des normes à la négociation d'entreprise, faisant reposer leur mise en œuvre sur des négociateurs plus ou moins outillés - qu'ils soient délégués du personnel, syndicaux, mandatés, etc. - lorsqu'ils existent.

\section{I.2. L'émergence de nouveaux acteurs...}

Dans le même temps, de nouveaux acteurs des relations professionnelles apparaissent, dépassant le seul trio dunlopien constitué par les organisations patronales, syndicales et l'État. Les acteurs institutionnels sont d'ailleurs eux-mêmes multiples et peuvent être analysés comme des structures aux intérêts hétérogènes à déconstruire selon les niveaux, les scènes et les arènes des relations professionnelles. Mais au-delà, c'est la complexification des objets de la régulation qui implique la montée en puissance d'experts en relations sociales (Cristofalo, 2009, 2013), en négociation, en appui aux comités d'entreprise, aux syndicats, aux employeurs, à l'État. D'autres acteurs entendent également s'imposer sur la scène sociale en jouant de leur capacité d'expertise comme le font de plus en plus les think tanks - et parmi eux ceux qui défendent les intérêts des entreprises et interviennent sur les normes d'emploi (Flocco, Rey, 2012). Les réseaux sociaux comptent de plus en plus dans les conflits, les mobilisations, les réformes. Sites internet, blogs de salariés, sites spécialisés sur les relations professionnelles, sur les salaires, ou encore sur les questions de santé au travail contribuent à la diffusion et au partage d'informations qui sont autant de ressources de pouvoir. En dehors et à côté du salariat, une hétérogénéité d'acteurs collectifs semblent intervenir de plus en plus dans le cours des régulations collectives. De multiples acteurs (ONG, associations, collectivités locales, etc.) se saisissent aujourd'hui d'enjeux propres aux relations professionnelles, tandis que d'autres secteurs (logement, environnement, formation, santé, etc.) sont investis par les acteurs traditionnels des relations professionnelles. De fait, sans imaginer que tout est nouveau - par exemple, des avocats travaillent depuis longtemps avec les confédérations syndicales ; le paritarisme 
dans le domaine de la formation n'est pas récent -, c'est peut-être même la notion d'acteur des relations professionnelles qui nécessite d'être réinterrogée. Les textes d'Aline Conchon, d'Alexandra Garabige et de Guy Bellemare et Louise Briand (dans ce numéro) explorent et analysent les jeux qui se jouent entre anciens et nouveaux acteurs. À partir d'enquêtes de terrain, ils donnent à voir la diversité des configurations et des situations que génère l'émergence d'acteurs s'impliquant dans la régulation.

\section{I.3. ... Et de nouveaux objets}

De nouveaux objets ou thématiques émergent dans ces configurations, dont les acteurs cherchent à s'emparer : la violence au travail, les restructurations, la discrimination... Le salariat se recompose, s'articule avec des formes d'entrepreneuriat, de sous-traitance et d'indépendance. Le travail se fait à distance, avec de nouvelles exigences de conciliation, de sécurité et d'égalité, de reconnaissance et d'engagement. Ces enjeux interpellent les organisations syndicales qui doivent continuer, par ailleurs, à traiter les problématiques plus classiques du salariat - la rémunération, la formation professionnelle, le respect des droits... - et gérer les dispositifs paritaires dans lesquels ils sont engagés. Les textes de Martine Gadille et Isabelle Schockaert, d'Hervé Champin, de Sophie Béroud et Jean-Michel Denis (dans ce numéro) s'intéressent surtout à l'acteur syndical dans ses rapports à des objets ou des enjeux a priori étrangers au champ des relations professionnelles ou à leur histoire propre : la propriété intellectuelle, l'établissement de normes au niveau européen, la perspective d'une organisation interprofessionnelle. Ces textes montrent comment les organisations s'emparent de ces sujets nouveaux et les intègrent, avec plus ou moins de succès, dans leurs pratiques organisationnelles et revendicatives.

Les articles regroupés dans ce numéro contribuent alors à enrichir toute une littérature qui s'est intéressée à la manière dont les acteurs des relations professionnelles ont réussi à se maintenir, parfois à progresser, sur la scène sociale et dans les entreprises, après de nombreuses morts annoncées. Cette capacité de résistance doit beaucoup à leur capacité d'innovation sociale alors que s'opère la lente mutation du paradigme fordiste et la fragilisation des cadres antérieurs de relations professionnelles, élaborés dans et pour ce paradigme.

\section{Processus d'innovation et changement en relations professionnelles : six cas de figures}

Les articles proposés ici ont pour point commun d'analyser, à partir de cas empiriques approfondis, des situations diverses de changements qui interpellent les acteurs des relations professionnelles. Plusieurs angles sont privilégiés qui permettent de saisir la complexité de la dynamique d'innovation. 


\section{II.1. Difficultés liées à l'émergence d'enjeux nouveaux}

Les recherches d'Aline Cochon - s'interrogeant sur les actionnaires comme nouveaux acteurs des relations professionnelles - et de Martine Gadille et Isabelle Schockaert - consacrées à la régulation de la propriété intellectuelle comme nouvel enjeu des relations professionnelles - mettent au jour les tensions ou difficultés que peuvent générer l'apparition ou la reconnaissance de thématiques ou d'espaces nouveaux, a priori extérieurs au champ des relations professionnelles. Aline Conchon montre en effet qu'en Europe, si le droit institutionnalise la représentation des salariés au sein des organes sociaux des entreprises (notamment dans le conseil d'administration ou de surveillance et l'assemblée générale des actionnaires), il peine à favoriser l'émergence d'une régulation conjointe entre actionnaires et élus du personnel. Pour autant, l'étude spécifique du cas français montre que les actionnaires s'imposent progressivement comme des acteurs légitimes et reconnus comme tels, notamment par les organisations syndicales. L'auteure conclut à l'existence des « apporteurs de capitaux» comme nouvel acteur du système des relations professionnelles et à la nécessité de prendre en compte ce dernier pour saisir la dynamique de régulation du travail et de l'emploi au niveau de l'entreprise. Martine Gadille et Isabelle Schockaert, quant à elles, explorent à partir d'une analyse des normes et règles juridiques, complétée d'études de cas empiriques, les tensions et conflits qui se jouent autour de la reconnaissance de la propriété intellectuelle dans l'entreprise et entre entreprises. Dans un contexte où la hiérarchie des normes applicable en la matière est donnée par le Code de la propriété intellectuelle et non (ou de manière marginale) par le Code du travail, l'encadrement juridique s'avère inapproprié pour prendre en compte la diversité des activités et situations d'innovation dans les entreprises. Les auteures pointent alors le besoin pour le syndicalisme de s'engager autour de cette problématique, de sorte que le droit puisse favoriser la reconnaissance des créations intellectuelles et inventions, en lien avec le travail et les conditions de travail.

\section{II.2. Des démarches ou projets innovants}

L'optique retenue par Sophie Béroud et Jean-Michel Denis dans leur recherche sur l'union syndicale Solidaires et par Alexandra Garabige dans son analyse de la participation dans une collectivité territoriale est tout autre. Elle consiste à analyser des situations dans lesquelles les acteurs des relations professionnelles cherchent à produire ou revendiquent la mise en place de démarches ou de projets innovants. Dans le cas de Solidaires, cette innovation fait référence au fait de bâtir son activité sur une démarche interprofessionnelle en se différenciant des autres organisations syndicales. Sophie Béroud et Jean-Michel Denis, au travers de la genèse de la structuration de Solidaires et d'une enquête approfondie (par questionnaires, 
entretiens, observations) auprès de ses militants, questionnent la capacité de l'union syndicale à maintenir son projet de transformation sociale. Les auteurs retracent la dynamique de construction tâtonnante de l'organisation, en présentant les évolutions, mais aussi les difficultés d'application concrète de la conception interprofessionnelle auxquelles se heurtent les militants. Au final, les auteurs montrent bien la difficulté d'expérimenter des formes innovantes d'organisation, en raison d'une structuration et de formes d'institutionnalisation qui peuvent peser sur l'activité de l'union syndicale. De son côté, la municipalité étudiée par Alexandra Garabige s'impose également de manière volontariste des modalités de démocratie participative. L'innovation tient à l'introduction de processus de participation des agents, mais aussi des usagers, destinés à aller au-delà des régulations sociales et politiques classiques. L'introduction de cette participation questionne les règles en place mais, comme dans le cas précédent, révèle la difficulté pour les acteurs de s'approprier les nouvelles procédures. Le bilan montre que ces démarches, dans leur mise en œuvre, sont façonnées en fonction des règles institutionnelles et organisationnelles qui existaient antérieurement et que les acteurs peinent à produire des transformations sociales.

\section{II.3. L'invention de nouvelles formes d'action}

Les analyses d'Hervé Champin d'une part et de Guy Bellemare et Louise Briand d'autre part révèlent au contraire la capacité des acteurs syndicaux à innover et inventer de nouvelles formes d'action, permettant d'enrayer la dynamique de fragilisation des systèmes de relations professionnelles. Hervé Champin montre, à partir de l'étude du dialogue social européen dans le secteur ferroviaire, que depuis les années 2000, les acteurs des relations professionnelles ont su créer des outils et des règles au niveau communautaire. L'innovation institutionnelle que constitue le dialogue social sectoriel européen permet aux acteurs de faire face et d'accompagner le processus d'européanisation de l'activité économique, même si, comme l'auteur le souligne, la dynamique positive à ce niveau retombe vers la fin des années 2000. Guy Bellemare et Louise Briand décrivent, quant à eux, la façon dont le mouvement syndical au Québec a su s'engager et s'imposer comme un acteur fort face à l'État, lui permettant d'influer dans la construction d'une politique sociale que sont les services de garde de la petite enfance. En retraçant le processus de construction depuis les années 1970 de ce mouvement social, ils montrent comment, sur le long terme, les syndicats ont su s'adapter en s'ouvrant et en créant des coalitions avec d'autres acteurs a priori extérieurs au champ des relations professionnelles (mouvement féministe, acteurs de l'économie sociale...). L'étude de cette innovation syndicale - consistant notamment à se saisir de problèmes 
relevant du champ politique - conduit les auteurs à proposer un nouveau cadre d'analyse pour penser les transformations dans la sphère des relations professionnelles.

Ainsi, dans les articles d'Aline Cochon et de Martine Gadille et Isabelle Schockaert, la dynamique d'innovation est à peine naissante. Les auteures pointent plutôt la nécessité pour les acteurs des relations professionnelles d'innover pour prendre en compte des évolutions qui leur sont extérieures. Sophie Béroud et Jean-Michel Denis d'une part, Alexandra Garabige d'autre part présentent au contraire le cas d'institutions qui s'imposent des innovations mais dont l'appropriation est contrariée par les routines organisationnelles antérieures. Les articles d'Hervé Champin et de Guy Bellemare et Louise Briand, enfin, montrent des innovations réussies, même si l'impact de ces processus reste à évaluer.

\section{Les innovations en relations professionnelles imposent-elles des innovations théoriques?}

Les articles rassemblés dans ce numéro s'attachent, nous l'avons vu, à décrire des processus d'innovation qui touchent trois dimensions des relations professionnelles : ses acteurs, ses objets et les espaces dans lesquels elles se déploient. Si l'on reprend la définition la plus communément utilisée qui définit les relations professionnelles comme «l'analyse des relations qui se nouent entre trois catégories d'acteurs, États, syndicats et patronats à propos des conditions de travail et de l'emploi salarié » (Béthoux, Jobert, 2004:261), on se focalise alors sur les règles qui gouvernent la relation d'emploi, les institutions impliquées dans ce processus et la dynamique des rapports de force entre les principaux acteurs et plus globalement sur la régulation collective et institutionnelle du travail et de l'emploi (Hyman, 2008). Il ne s'agit alors pas d'étudier toutes les relations d'emploi mais celles régulées par des arrangements institutionnels, pas d'analyser l'ensemble des acteurs mais uniquement les acteurs collectifs.

Les relations professionnelles, en tant que modèle explicatif, laissent ainsi hors champ les dimensions informelles et individuelles du travail. La portée heuristique du modèle se trouve désormais affaiblie par le mouvement de distance croissant des individus avec les rôles sociaux que les institutions (dont les syndicats) avaient coutume de leur assigner, mouvement dont les sociologues contemporains veulent rendre compte avec le concept de dé-institutionnalisation (Lallement, 2004 ; Conchon et al., 2011). Les grandes institutions héritées des années 1950 sont ébranlées par une plus grande pluralité de logiques et de modes de fonctionnement des acteurs. Les travaux menés, depuis Dunlop, au sein de la communauté des chercheurs en relations professionnelles ne peignent d'ailleurs pas une réalité 
stable et figée mais décrivent des situations hétérogènes et complexes à des échelles diverses. Jusqu'à maintenant, ces chercheurs considéraient la notion de système de relations professionnelles suffisamment plastique pour s'adapter à des réalités sociales très différentes (Mias, 2012). Les processus d'individualisation à l'œuvre entraînent-ils un divorce entre acteurs et institutions dans le champ du travail, rendant inopérante l'approche en termes d'acteurs collectifs ? Les évolutions en cours remettent-elles en question l'approche dunlopienne en termes de système de relations professionnelles au point d'imposer des innovations théoriques seules à même de comprendre les nouvelles réalités sociales? L'article d'Aline Conchon et celui de Guy Bellemare et Louise Briand attaquent de front ces interrogations.

Pour Aline Conchon, il convient de distinguer, dans le camp des directions d'entreprises, deux acteurs aux logiques et intérêts distincts : les apporteurs de capitaux ou actionnaires et les dirigeants. Bien que Dunlop les ait explicitement exclus, les apporteurs de capitaux sont pour elle un nouvel acteur des relations professionnelles. Guy Bellemare, dans un article antérieur (2000), avait déjà ajouté un autre acteur : les utilisateurs finaux (end users), comme par exemple les clients. Dans l'article qu'il propose ici avec Louise Briand, les usagers des services de garde de la petite enfance, les parents, apparaissent également comme de possibles utilisateurs finaux. Cette question est aussi posée par Alexandra Garabige quand elle décrit comment la participation des usagers dans une collectivité territoriale perturbe le fonctionnement routinier des relations professionnelles. Comme acteurs des relations professionnelles, les parents québécois sont plus organisés que les habitants d'une commune : ils gèrent directement les centres de petite enfance associatifs et, à ce titre, sont aussi employeurs. D'une certaine manière, le salarié « inventeur » de Martine Gadille et d'Isabelle Schockaert qui réclame la reconnaissance de ses droits de propriété intellectuelle n'est plus dans le cadre strict du lien de subordination à son employeur. Ce qui ressort de ce numéro est, à l'évidence, un plus grand flou des rôles sociaux joués par les acteurs des relations professionnelles. Peut-on continuer à les ériger en acteurs collectifs à part entière ? Les actionnaires, dont les intérêts ne coïncident pas simplement avec ceux des dirigeants d'entreprises ont-ils pour autant une logique réellement distincte de ces derniers ? La place des usagers et le flou des rôles patronaux dans le secteur public sont patents mais n'est-ce pas lié justement aux spécificités de ce secteur particulier? Doit-on en inférer une remise en cause du rôle des acteurs dans le secteur privé ? Pour répondre à ces questions, il faut certainement suivre l'invitation faite par Aline Conchon aux chercheurs en relations professionnelles à étudier plus systématiquement les nouvelles catégories d'acteurs.

Au-delà des acteurs, les auteurs questionnent également la pertinence des espaces dans lesquels ceux-ci agissent. De manière ambitieuse, 
Guy Bellemare et Louise Briand appellent à un dépassement du concept de système de relations professionnelles et à son remplacement par la notion de région de rapport de travail empruntée à Giddens afin de mieux prendre en compte le champ politique de la vie, terme également repris de Giddens qui se rapproche de ce qu'en France on appelle la société civile. La notion de région de rapport de travail, qui n'a rien de géographique mais est socialement construite, renvoie à la structuration de l'action dans un espace et un temps définis par les acteurs eux-mêmes. Les innovations sociales décrites par les auteurs, les stratégies d'alliances des syndicats avec des mouvements sociaux et des acteurs de la société civile (parents, directrices de crèche par exemple) exigent selon eux des innovations théoriques. Sans aller aussi loin, la question posée en filigrane par l'ensemble des articles est celle des frontières entre les différents espaces des rapports sociaux, en d'autres termes de la dé-différenciation de pratiques sociales considérées jusque-là comme distinctes. Les innovations syndicales québécoises font écho à celles qu'entendent promouvoir les syndicats regroupés au sein de Solidaires. Sophie Béroud et Jean-Michel Denis montrent comment la volonté de ce syndicat d'intégrer une dimension sociétale pourrait permettre de dépasser la crise de la syndicalisation française mais aussi que le passage à un stade différent de l'organisation avive des tensions : comment concilier le développement au niveau interprofessionnel avec l'action syndicale de proximité difficilement nouée par les militants dans les entreprises?

Mobilisant un autre registre théorique, Aline Conchon s'accorde avec les tenants de la théorie de la complémentarité institutionnelle pour considérer que les deux systèmes qu'elle étudie, le système de relations professionnelles et celui de gouvernement d'entreprise sont interdépendants, encastrés. Les organes sociaux de l'entreprise constituent un nouvel espace de régulation commun aux actionnaires et aux salariés que ces derniers peinent pour l'instant à investir. Hervé Champin souligne bien aussi que le passage du niveau national au niveau européen est plus qu'un simple changement d'échelle pour les acteurs syndicaux et patronaux du secteur des chemins de fer. Les processus d'élaboration des normes et d'alliances entre les acteurs de ce niveau ne procèdent pas du déploiement des règles du jeu constatées dans les différents systèmes nationaux mais composent un nouvel espace de régulation. La reconnaissance de la propriété intellectuelle peut également être vue comme un processus conduisant à encastrer les champs du travail et de l'économie de la connaissance. L'innovation sociale serait finalement une entrée pertinente pour aborder la pluralité des dynamiques de recomposition du champ des relations professionnelles et les nouvelles formes d'encastrement avec d'autres espaces du champ social qui en découlent. 


\section{Conclusion}

Ces recherches mettent ainsi au jour la diversité des démarches et des enjeux rompant avec le schéma classique des relations professionnelles et conduisant les acteurs, en particulier syndicaux, à définir et redéfinir leur stratégie et leur positionnement. L'hétérogénéité des situations analysées permet de questionner la capacité des acteurs à innover, qu'il s'agisse de se saisir de nouvelles règles institutionnelles, de nouveaux sujets ou de les créer. Dans cette période de changement, on observe à la fois une série d'obstacles mais aussi l'émergence de voies nouvelles dans lesquelles les acteurs des relations professionnelles s'engagent ou pourraient s'engager pour participer pleinement à la production d'innovations sociales. Il faudrait alors poursuivre l'analyse notamment en évaluant l'impact de ces processus innovants. 


\section{Références bibliographiques}

Bellemare G. (2000), " End Users: Actors in the Industrial Relations System? ", British Journal of Industrial Relations, vol. 38, $n^{\circ} 3$, September, p. 383-405.

Bellemare G., Briand L. (2013), "La syndicalisation des services de garde au Québec : à pratiques innovatrices, des concepts nouveaux ", La Revue de I'IRES, $\mathrm{n}^{\circ} 75$, p. 117-141.

Béroud S., Denis J.-M. (2013), " Le développement interprofessionnel de Solidaires : entre volonté d'expérimentation et reproduction du "modèle" confédéral ", La Revue de I'IRES, n 75, p. 67-92.

Béthoux É., Jobert A. (2004), " Regards sur les relations professionnelles en Europe et en Amérique du Nord : évolutions et perspectives ", Sociologie du travail, $\mathrm{n}^{\circ} 46$, p. $261-270$.

Champin H. (2013), « Dialogue social européen et innovation : l'exemple du secteur des chemins de fer ", La Revue de I'IRES, n²7, p. 143-167.

Conchon A., Dressen M., Rey F. (2011), « Désinstitutionnalisation des relations professionnelles ? ", Revue multidisciplinaire sur l'emploi, le syndicalisme et le travail (REMEST), vol. 6, n 1, p. 125-146.

Conchon A. (2013), "L'actionnaire, nouvel acteur des relations professionnelles au niveau de l'entreprise ? ", La Revue de I'IRES, n 75 , p. 43-65.

Cristofalo P. (2009). «L'institutionnalisation d'une fonction d'expertise et de conseil auprès des élus du personnel », Cahiers internationaux de sociologie, vol. CXXVI, p. 81-98.

Cristofalo P. (2013). « Dynamiques et limites de l'autonomisation de l'expertise auprès des CHSCT », La Revue de l'IRES, n 74, p.127-151.

Flocco G., Rey F. (2012), « Une analyse relationnelle des clubs et "think tanks" patronaux. Des interfaces aux frontières de l'espace patronal », in Amossé T., Flocco G., Lefèvre J., Pernot J.-M., Petit H., Rey F., Tallard M., Tuchszirer C., Vincent C., Les organisations patronales en France. Continuités et mutations des formes de représentation du patronat, Rapport de recherche IRES, p.159-192.

Gadille M., Schockaert I. (2013), « Reconnaissance des créations de salariés et régulation de la propriété intellectuelle : un enjeu émergent des relations professionnelles ", La Revue de l'IRES, n 75, p. 15-42.

Garabige A. (2013), « La participation des usagers et des agents dans une collectivité locale : entre innovation et permanence du modèle d'administration publique », La Revue de I'IRES, $n^{\circ} 75$, p. 93-115.

Groux G. (2005), « L'action publique négociée. Un nouveau mode de régulation ? Pour une sociologie politique de la négociation ", Négociations, $\mathrm{n}^{\circ} 3$, p. $57-70$.

Hyman R. (2008), "What's the Point of Industrial Relations? ", Statement by the British Universities Industrial Relations Association (BUIRA), BUIRA Conference, 26-28 June, University of the West of England, Bristol.

Lallement M. (2004), « Penser les institutions: paradigmes d'hier, débats d'aujourd'hui », Sociologia del lavoro, supplément au $n^{\circ} 93$, p. 62-75.

Mahoney J., Thelen K. (2010), « A Theory of Gradual Institutional Change », in Mahoney J., Thelen K. (eds), Explaining Institutional Change: Ambiguity, Agency, and Power, New York, Cambridge University Press, p. 1-37.

Mias A. (2012), John T. Dunlop, Industrial Relations Systems. Les règles au coeur des relations de travail, Paris, Ellipses. 